\title{
On the intraatomic Coulomb and exchange energies in degenerate narrow bands
}

\author{
G. Allan \\ Laboratoire de Physique des Solides (*), I.S.E.N., \\ 3, rue François-Baës, 59 Lille, France \\ J. Friedel \\ Laboratoire de Physique des Solides (*), Université Paris-Sud, 91405 Orsay, France \\ and C. M. Sayers \\ AERE, Harwell, Oxfordshire, England
}

(Reçu le 25 février 1980, accepté le 20 avril 1980)

\begin{abstract}
Résumé. - Dans les bandes étroites dégénérées étudiées en liaisons fortes, les fonctions de Wannier des bandes partielles ne peuvent être utilisées pour calculer les interactions de Coulomb intraatomiques, parce que chacune d'elles met en jeu plusieurs fonctions atomiques, sur des sites différents. Dans l'approximation (de Hartree-Fock) qui utilise un déterminant fait de fonctions de Bloch, le changement d'énergie de Coulomb intraatomique entre les atomes libres et le métal paramagnétique varie ainsi de façon parabolique avec le nombre d'états atomiques occupés, et non avec le nombre d'états occupés dans chaque bande partielle. Ceci justifie des traitements précédents de ce problème et corrige un travail récent de Thalmeier et Falicov. Le changement d'énergie d'échange intraatomique est également explicité.
\end{abstract}

\begin{abstract}
In degenerate narrow bands studied in the tight binding limit, Wannier functions of partial bands cannot be used for computing intraatomic Coulomb interactions, because each of them involves several atomic states, on different sites. In the (Hartree-Fock) approximation using one determinant made of Bloch functions, the change in intraatomic Coulomb energy from free atoms to paramagnetic metal thus varies parabolically with the numbers of occupied atomic states, and not with the number of occupied states in each partial band. This justifies earlier treatments of the problem and corrects a recent work by Thalmeier and Falicov. The change in intraatomic exchange energy is also explicitly given.
\end{abstract}

1. Wannier versus atomic states in degenerate bands. - In a recent work [1], Thalmeier and Falicov have computed the intraatomic Coulomb energy in a degenerate narrow band. They have equated the Coulomb energy of two electrons, of opposite spins, in the same Wannier state of the metal, to the Coulomb energy $U$ of two electrons in the same atomic state in the free atom. With this approximation, these authors have computed the contribution to lowest order (one determinant mode of Bloch functions) of this energy to cohesion. They have shown that this contribution is sensitive to the crystal structure and also differs

(*) L.A. au C.N.R.S. much from an earlier estimate made by one of us [2,3] and used in more recent studies of second order terms $[4,5,6]$. Practical applications are to degenerate $\mathrm{d}$ bands of transitional metals studied in the simplest tight binding limit.

We wish to point out that the Coulomb energy of two electrons in the same Wannier state is usually different from $U$, and indeed varies with the subband considered as well as with the way Wannier states are defined [7]. The reason is simply that, in a degenerate band studied in tight binding, a Wannier state of one subband extends over more than one atomic site [8]. Thalmeier and Falicov's treatment if furthermore inconsistent in that they do not use Wannier functions to compute the total Coulomb energy in the metallic 
state, but only that part which is in excess of the Coulomb energy in free atoms.

2. Intraatomic Coulomb energy using atomic states. A more correct way of computing the Coulomb energy is to introduce directly the energy $U$ of electrons sitting in atomic states on the same site, and to evaluate the change due to $U$, in the simplest HartreeFock scheme, when one goes from free atoms to the paramagnetic metal. This is what was done in our earlier treatment, which explicitly used the random uncorrelated distribution of the electrons over Bloch states. We repeat here the argument, using a more explicit formulation.

We write the total function $|\Psi\rangle$ of the metal as a determinant of Bloch functions with spatial part

$$
|\mathbf{k}\rangle=\sum_{\mathbf{R}} \mathrm{e}^{i \mathbf{k} \mathbf{R}} \sum_{i} a_{\mathbf{k}}^{i}\left|\mathbf{R}_{i}\right\rangle
$$

The summation $\mathbf{R}$ is over lattice sites. The summation $i$ is over the $n$ degenerate atomic states $(n=5$ for $\mathrm{d}$ bands). A straightforward and classical computation gives, for the total Coulomb energy between the $d$ electrons in the metal :

$$
\begin{aligned}
A=\left\langle\Psi\left|\sum_{l>m} \frac{1}{r_{l m}}\right| \Psi\right\rangle \\
=\sum_{\mathbf{k}_{\mathrm{bc} . \mathbf{k}}^{\prime} \mathbf{k}_{\mathrm{occ}}}\left[2\left\langle\mathbf{k}(1) \mathbf{k}^{\prime}(2)\left|\frac{1}{r_{12}}\right| \mathbf{k}(1) \mathbf{k}^{\prime}(2)\right\rangle-\right. \\
\left.\quad-\left\langle\mathbf{k}(1) \mathbf{k}^{\prime}(2)\left|\frac{1}{r_{12}}\right| \mathbf{k}^{\prime}(1) \mathbf{k}(2)\right\rangle\right]
\end{aligned}
$$

$1,2, l, m$ are indices denoting electrons. $\mathbf{k}_{\mathrm{occ}}$ and $\mathbf{k}_{\mathrm{occ}}^{\prime}$ denote states $\mathbf{k}$ and $\mathbf{k}^{\prime}$ that are occupied, with energies below the Fermi level of the metal. The factors 2 and 1 come from the spin degeneracy; the first terms are the classical Coulomb terms and the second ones are the exchange terms.
We denote, for any $i, j$ on any site $\mathbf{R}$ :

$$
\begin{aligned}
& \left\langle\mathbf{R}_{i}(1) \mathbf{R}_{j}(2)\left|\frac{1}{r_{12}}\right| \mathbf{R}_{i}(1) \mathbf{R}_{j}(2)\right\rangle=U \\
& \left\langle\mathbf{R}_{i}(1) \mathbf{R}_{j}(2)\left|\frac{1}{r_{12}}\right| \mathbf{R}_{j}(1) \mathbf{R}_{i}(2)\right\rangle=U \delta_{i j}+J\left(1-\delta_{i j}\right),
\end{aligned}
$$

and neglect all the other Coulomb terms. If $N$ is the total number of sites, $A$ then reduces to

$$
A=N U\left(2 \sum_{i} Z_{i} \sum_{j} Z_{j}-\sum_{i} Z_{i}^{2}\right)-N J \sum_{i, j \neq i} Z_{i} Z_{j}
$$

Here

$$
Z_{i}=\sum_{\mathbf{k}_{\mathrm{occ}}}\left|a_{\mathbf{k}}^{i}\right|^{2}
$$

is the number of electrons with one spin direction in the atomic orbital $i$ on site $\mathbf{R}$. It is such that, if there are $p$ electrons per atom in the paramagnetic metal,

$$
2 \sum_{i} Z_{i}=p .
$$

Equation (1) can also be written

$$
A=N U\left(2 \sum_{i, j \neq i} Z_{i} Z_{j}+\sum_{i} Z_{i}^{2}\right)-N J \sum_{i, j \neq i} Z_{i} Z_{j}
$$

It has a simple physical meaning, which was used directly previously $[4,5,6]$ :

- The first term is the intraatomic Coulomb interaction of electrons in different atomic orbitals $i$, $j$ when distributed at random over $N$ sites with average numbers $Z_{i}, Z_{j}$ per site.

- The third term is the corresponding intraatomic exchange energy.

- The second term is the special intraatomic Coulomb energy of electrons in the same atomic orbital, when correction has been made of the corresponding exchange term, or equivalently when the Pauli principle has been taken into account.

The corresponding terms in $N$ free (magnetic) atoms can be written in the same way $\left({ }^{1}\right)$ :

$$
\begin{array}{lll}
B=N\left(\frac{U}{2} p(p-1)-\frac{J}{2} p(p-1)\right), & \text { if } & p<n(=5 \text { for d bands }) \\
B=N\left(\frac{U}{2} p(p-1)-\frac{J}{2}\{n(n-1)+(p-n-1)\}\right) & \text { if } & p>n .
\end{array}
$$

Finally, the contribution to the cohesive energy per atom of the Coulomb and exchange (Hartree-Fock) terms is

$$
\Delta E_{\mathrm{c}}=\frac{B-A}{N}=-U \sum_{i} Z_{i}\left(1-Z_{i}\right)-\alpha J
$$

where

$$
\begin{aligned}
& \alpha=\frac{p(p-1)}{2}-\sum_{i, j \neq i} Z_{i} Z_{j} \\
& \text { if } \quad 0 \leqslant p \leqslant n \\
& \alpha=\frac{1}{2}\{n(n-1)+(p-n)(p-n-1)\}-\sum_{i, j \neq i} Z_{i} Z_{j} \quad \text { if } \quad n \leqslant p \leqslant 2 n \text {. }
\end{aligned}
$$

( $\left.{ }^{1}\right)$ For non integral values of $p$, a correction should be made to the exchange term [5]. 
Thus the Coulomb part of $\Delta E_{\mathrm{c}}$ varies parabolically with the average population $Z_{i}$ of each atomic orbital $i$, and not with the population of each subband, as stated by Thalmeier and Falicov [1].

It is also easily deduced from these formulae that $\Delta E_{\mathrm{c}}=0$ for a completely empty band (all $Z_{i}$ 's $=p=0$ ) as well as for a completely full band (all $Z_{i}^{\prime}$ 's $=1$ and $p=2 n$ ). The Coulomb part in $\Delta E_{\mathrm{c}}$ is always negative, with a broad minimum for a nearly half filled band $(p=n)$; the exchange part in $\Delta E_{\mathrm{c}}$ has an acute minimum for a half filled band $(p=n)$.

In alternant structures [8] where the band structure is symmetrical in energy, one has $Z_{i}(p)=1-Z_{i}(2 n-p)$ and thus $Z_{i}(n)=\frac{1}{2}$. The minimum of $E_{\mathrm{c}}$ is then exactly for a half filled band $(p=n)$ and is equal to

$$
E_{\mathrm{c} \min }=-U \frac{n}{2}-J \frac{n(n-1)}{4}
$$

Even in non alternant structures, $\Delta E_{\mathrm{c}}$ does not deviate much from these characteristics.

Indeed numerical computations [9] have shown that, in the closepacked structures, the various $d$ orbitals have nearly equal populations for most filling of the $d$ band. In our previous analysis [4], we have used therefore the simple approximation :

$$
Z_{i}=\frac{p}{2 n} \quad(n=5 \text { for } \mathrm{d} \text { bands })
$$

Then $\Delta E_{\mathrm{c}}(p)$ is symmetrical with respect to $p=n$, and can be written

$$
\begin{array}{llrl}
\Delta E_{\mathrm{c}}=-\frac{U}{2} p\left(1-\frac{p}{2 n}\right)-\frac{J}{2}\left\{p(p-1)-\frac{n-1}{2 n} p^{2}\right\} & \text { for } & p<n \\
\Delta E_{\mathrm{c}}=-\frac{U}{2} p\left(1-\frac{p}{2 n}\right)-\frac{J}{2}\left\{(2 n-p)(2 n-p-1)-\frac{n-1}{2 n}(2 n-p)^{2}\right\} & \text { for } & p>n .
\end{array}
$$

We should point out that a numerical error occurred in the value of the coefficient of the exchange term $J$ in references [4] to [6] which however does not alter the general variation of the exchange term nor its minimum (negative) value. Also approximation (2) is less valid for nearly full or nearly empty bands.

\section{References}

[1] Talmeier, P. and Falicov, L. M., Phys. Rev. B 20 (1979) 4637.

[2] Friedel, J., J. Physique Radium 16 (1958) 829.

[3] Friedel, J., in the Physics of Metals. I. Electrons, edited by J. M. Ziman (Cambridge University Press, Cambridge) 1969 , p. 340

[4] Friedel, J., Ann. Phys. 1 (1976) 257.

[5] Friedel, J. and SAYers, C. M., J. Physique 38 (1977) 697; J. Physique Lett. 38 (1977) L-263 ; 39 (1978) L-59.

[6] Friedel, J., J. Magnet. Mat., under press.
[7] Blount, E. I., Solid State Phys. 13 (1962) 305.

[8] Friedel, J., J. Physique 39 (1978) 651, 671.

[9] Asdente, M. and Delitala, M. D., Phys. Rev. 163 (1967) 497 ; Ducastelle, F. and Cyrot-Lackmann, F., J. Phys. Chem. Solids 31 (1970) 1295 ;

Desjonquères, M. C. and Cyrot-Lackmann, F., J. Chem. Phys. 64 (1976) 3707 ;

Anderson, O. K., Physica B 91 (1977) 317. 